\title{
BMJ Open Complementary vaccination protocol with dendritic cells pulsed with autologous tumour lysate in patients with resected stage III or IV melanoma: protocol for a phase II randomised trial (ACDC Adjuvant Trial)
}

Laura Ridolfi, ${ }^{1}$ Francesco de Rosa, ${ }^{1}$ Laura Fiammenghi, ${ }^{1}$ Massimiliano Petrini, ${ }^{1}$ Anna Maria Granato, ${ }^{1}$ Valentina Ancarani, ${ }^{1}$ Elena Pancisi, ${ }^{1}$ Valentina Soldati, ${ }^{1}$ Serena Cassan, ${ }^{1}$ Jenny Bulgarelli, ${ }^{1}$ Angela Riccobon, ${ }^{1}$ Giorgia Gentili, ${ }^{2}$ Oriana Nanni, ${ }^{2}$ Massimo Framarini, ${ }^{3}$ Francesca Tauceri, ${ }^{3}$ Massimo Guidoboni ${ }^{1}$

To cite: Ridolfi L, de Rosa F, Fiammenghi L, et al. Complementary vaccination protocol with dendritic cells pulsed with autologous tumour lysate in patients with resected stage III or IV melanoma: protocol for a phase II randomised trial (ACDC Adjuvant Trial). BMJ Open 2018;8:e21701. doi:10.1136/ bmjopen-2018-021701

- Prepublication history for this paper is available online. To view these files, please visit the journal online (http://dx.doi org/10.1136/bmjopen-2018021701).

LR and FR contributed equally.

Received 15 January 2018 Revised 29 May 2018 Accepted 10 July 2018

Check for updates

(C) Author(s) (or their employer(s)) 2018. Re-use permitted under CC BY-NC. No commercial re-use. See rights and permissions. Published by BMJ.

For numbered affiliations see end of article.

Correspondence to

Dr Laura Ridolfi;

laura.ridolfi@irst.emr.it

\section{ABSTRACT}

Introduction Surgery is one of the treatments of choice for patients with a single metastasis from melanoma but is rarely curative. Such patients could potentially benefit from consolidation immunotherapy. Vaccination with dendritic cells (DCs) loaded with tumour antigens elicits a tumourspecific immune response. In our experience, patients who developed delayed type hypersensitivity (DTH) after DC vaccination showed a median overall survival (OS) of 22.9 monthsvs4.8 months for DTH-negative cases. A phase II randomised trial showed an advantage $\mathrm{OS}$ of a $\mathrm{DC}$ vaccine over a tumour cell-based vaccine (2-year OS 72\% vs $31 \%$, respectively). Given that there is no standard therapy after surgical resection of single metastases, we planned a study to compare vaccination with DCs pulsed with autologous tumour lysate versus follow-up.

Methods and analysis This is a randomised phase II trial in patients with resected stage III/IV melanoma. Assuming a median relapse-free survival (RFS) of 7.0 months for the standard group and 11.7 months for the experimental arm (HR 0.60), with a two-sided tailed alpha of $0.10,60$ patients per arm must be recruited. An interim futility analysis will be performed at 18 months. The DC vaccine, produced in accordance with Good Manufacturing Practice guidelines, consists of autologous DCs loaded with autologous tumour lysate and injected intradermally near lymph nodes. Vaccine doses will be administered every 4 weeks for six vaccinations and will be followed by 3 million unit /day of interleukin-2 for 5 days. Tumour restaging, blood sampling for immunological biomarkers and DTH testing will be performed every 12 weeks. Ethics and dissemination The protocol, informed consent and accompanying material given to patients were submitted by the investigator to the Ethics Committee for review. The local Ethics Committee and the Italian Medicines Agency approved the protocol (EudraCT code no.2014-005123-27). Results will be published in a peerreviewed international scientific journal.

Trial registration number 2014-005123-27.
Strengths and limitations of this study

- This randomised study will evaluate whether specific immunisation against tumour cells improves relapse free and overall survival in resected stage III/IV melanoma compared with standard follow-up.

The therapy used has a very low toxicity profile.

- The trial aims to identify predictive immunological markers that could help to select patients who are most likely to benefit from adjuvant immunotherapy.

- As the study is based on a relatively rare tumour, its main limitation could be difficulties encountered with patient accrual.

- The cell factory at our institute can guarantee treatment for up to 78 patients over a period of 13 months, which is compatible with the statistical calculation made for this study.

\section{INTRODUCTION}

About $15 \%$ of patients with melanoma present with metastases, while in the remaining $85 \%$ metastatic disease is diagnosed after the diagnosis of the primary lesion. The 5-year survival rate of patients with metastatic disease is only $10 \%$ but almost doubles $(19 \%)$ in patients whose metastases are limited to soft tissues (skin, lymph nodes). ${ }^{1}$ Surgery or intensive local radiation is the treatment of choice for patients with a single metastasis but is seldom curative. Fiveyear survival rates of $20 \%-25 \%$ have been reported after surgical resection of a solitary metastasis, with similar survival whether the solitary metastasis is in the skin, lymph nodes, liver, alimentary tract or brain. ${ }^{2}$ The potential benefit from surgery was also seen in Sosman 
et $a l$ s study in 64 out of 77 carefully selected patients who underwent complete resection of all metastatic disease. The median duration of RFS and overall survival (OS) at a median follow-up of 5 years was 5 and 21 months, respectively. ${ }^{3}$ Overall, 3- and 4-year survival rates were $36 \%$ and $31 \%$, respectively, although late relapses were also observed after this time. Howard et al, analysing data from the Multicenter Selective Lymphadenectomy Trial (MSLT-I) for patients undergoing surgical treatment \pm systemicmedical treatment for metastatic disease, reported an advantage for any M1 metastatic stage (a, b or c) from surgery over medical treatment alone. ${ }^{4}$ In 2011, Wasif et al published a registry outcome analysis of patients who underwent surgery for stage IV melanoma, reporting a 2-year OS of almost $30 \%$ for stage M1c and around $40 \%$ for Mla. ${ }^{5}$ However, the most interesting data come from the phase III randomised Malignant Melanoma Active Immunotherapy Trial for Stage IV disease conducted at John Wayne Cancer Institute. This trial was initially conceived to test the efficacy of an allogeneic whole-cell vaccine (Canvaxin) plus BCG versus placebo plus BCG after complete resection of stage IV melanoma. Although there was no difference between treatment arms, a substantially longer and surprisingly high 5-year survival rate was observed for placebo-arm patients (39.6\% vaccine vs $44.9 \%$ placebo) ${ }^{6}$ Given the advent of newer and better systemic therapies, ${ }^{78}$ we believe that the role of surgical resection should be strongly considered as part of the treatment paradigm. ${ }^{59}$

Vaccination with dendritic cells (DCs) loaded with tumour antigens has been largely shown to elicit tumour-specific immune responses potentially capable of killing cancer cells without inducing meaningful side effects. DCs are antigen-presenting cells widely distributed in almost all tissues of the body that play a central role in the activation and regulation of the immune response. ${ }^{10}{ }^{11}$ As cancer progresses, tumour cells acquire the ability to either evade the immune response by selecting immunogenic variants (cancer immunoediting) ${ }^{12}$ and/or producing immunosuppressive cytokines and other biologically active substances that strongly influence the ability of DCs to prime and sustain effective immune responses. ${ }^{1314}$ In 1996, Schadendorf's group was the first to test the feasibility of a vaccination strategy in patients with melanoma. The authors aimed to restore DC function by differentiating them and loading them with tumour antigens ex vivo in an attempt to overcome the effects of a DC-tolerising tumour microenvironment. ${ }^{15}$ Since this first experience, it has been estimated that over 1000 patients with different tumours have been treated with vaccines using different starting cells and differentiation/maturation protocols, and different antigen sources and administration routes. ${ }^{16}{ }^{17}$ Since 2001 our institute has conducted several phase II clinical studies on patients with advanced melanoma using autologous DCs loaded with autologous tumour lysate (ATL)/homogenate matured with a cytokine cocktail. To date, $55.5 \%$ of evaluable patients have obtained a clinical benefit (partial response+stable disease). In our experience, the patients who developed antitumour immunity after vaccination experienced a better clinical outcome. In particular, we observed that patients developing delayed type hypersensitivity (DTH) against ATL or keyhole limpet haemocyanin (KLH) after at least four courses of the vaccine showed a HR of $0.54 .{ }^{18-21}$ In patients experiencing a clinical benefit from DC vaccination, regulatory $\mathrm{T}$ (Treg) lymphocytes showed a decrease after 5 days of low-dose interleukin-2 (IL-2) $(-33.8 \%),{ }^{20}$ whereas Tregs in patients in progression increased by $388.9 \%$.

Many patients with metastatic melanoma enjoy brief periods of no evidence of disease (NED) after surgical resection of metastases and/or radiation therapy and/ or systemic therapy, and such patients could potentially benefit from an effective, non-toxic, consolidation immunotherapy. Dillman et al observed an important advantage in OS from a DC-based vaccine in patients with minimal residual disease compared with tumour cells (2-year survival $72 \%$ vs $31 \%$, respectively), and a very good toxicity profile. ${ }^{22}{ }^{23}$ The results are eagerly awaited of the international phase 3 Bristol Meyer Squibb (BMS) CA 209-238 trial in patients with resected stage IIIC/IV melanoma randomly assigned to nivolumab or ipilimumab $10 \mathrm{mg} / \mathrm{kg}$ as adjuvant therapy after radical surgery. The study concluded enrolment while some patients were still undergoing treatment.

Given that there is still no standard therapy for patients with no evidence of disease (NED) after surgical resection of a single metastasis, and taking into account that immunotherapy is more effective in minimal residual disease, we designed a randomised phase II study in patients with NED after surgery for stage IV or metachronous stage III melanoma to evaluate vaccination with DCs pulsed with autologous tumour homogenate+low-dose IL-2 SC (subcutaneously) versus follow-up. We decided to maintain the treatment comprising low-dose IL-2 on the basis of our long-standing positive experience with the schedule and our published data on Tregs.

\section{METHODS AND ANALYSIS}

\section{Investigational product}

Since 2001, the Cell Therapy Laboratory (Laboratorio di Terapie Cellulari) of our institute (IRST IRCCS) has been producing a therapeutic vaccine composed of autologous DCs pulsed with ATL or homogenate for patients with metastatic melanoma or kidney cancer. The vaccine is prepared in accordance with two largely overlapping protocols.

\section{Freshly prepared vaccine}

Each vaccine dose is prepared from patients' monocytes obtained by leucapheresis. After leucapheresis, a part of the monocytes are cultured and the remainder is cryopreserved in aliquots for the manufacture of other vaccine doses. Monocytes are cultured for 6 days in serum-free, Good Manufacturing Practice (GMP)-certified medium 
supplemented with granulocyte-macrophage colony stimulating factor (GM-CSF) and IL-4 to obtain immature dendritic cells (iDCs). iDCs are then pulsed with autologous lysate or homogenate prepared from surgically removed tumour lesions and matured for 48 hours with a cytokine cocktail (tumor necrosis factor alfa, IL-1 $\beta$, IL-6, and prostaglandin E2). Mature DCs (mDCs) are then collected, washed, counted and resuspended in sterile saline (total $7-15 \times 10^{6}$ cells) for immediate intradermal injection into patients.

\section{Cryopreserved vaccine}

Vaccine is produced from the whole leucapheresis product according to the previously described protocol. After the maturation step, pulsed mDCs are collected, washed, counted, resuspended in sterile saline, aliquoted (total $7-15 \times 10^{6}$ cells) and cryopreserved by automated freezing. When needed, they are thawed, washed, resuspended in saline and immediately administered intradermally.

\section{OBJECTIVES}

Primary endpoint is relapse-free survival (RFS), that is, time from the date of randomisation to the date of the first relapse or the date of death from any cause or the date of the last restaging in non-relapsed patients.

Secondary endpoints are as follows:

- OS measured from the date of randomisation until the date of death from any cause or the last date on which the patient was known to be alive.

- In vivo and in vitro immunomonitoring.

- Toxicity.

- Prognostic and predictive markers of response.

- Immunologic response.

\section{TRIAL DESIGN}

\section{Statistical considerations}

This study is a randomised phase II trial (1:1 allocation ratio) in patients with resected stage III/IV melanoma. On the basis of literature data, we assume that the standard group will have a median RFS of 7.0 months. With a two-sided tailed alpha of 0.10 and a power of $80 \%$, assuming a median RFS of 11.7 months in the experimental arm (HR 0.60), it will be necessary to recruit 60 patients per arm over a period of 24 months and to have a follow-up of 12 months. Within the context of data monitoring activities, an interim futility analysis will be performed at 18 months according to the Bayesian approach proposed by Fayers et al (to control the safety). The randomisation list will be stratified by stage (III, IV M1a-b or IV M1c), and time from primitive tumour to first metastasis ( $\leq 2$ years vs $>2$ years). Four randomisation lists will be defined, one for each stratum.

\section{Study phases}

After surgery and tumour tissue collection, performed in accordance with GMP regulations and current standard operating procedures (SOPs) of the institute's Somatic
Cell Therapy (SCT) Laboratory, informed consent will be obtained from patients. A screening phase will follow to determine trial eligibility, after which patients will be randomised to either the vaccine arm or the control $\mathrm{arm}$. The former will enter the leucapheresis and vaccine manufacturing phase and the latter will begin clinical and radiological follow-up. Patient tumour tissue will be stored in the therapeutic biobank of the SCT Laboratory until needed.

\section{Vaccine arm}

Patients will undergo leucapheresis on day 9, and the vaccine will be prepared according to current SOPs included in the 'Product Specification File' (on GMP authorisation by the Italian Medicines Agency (AIFA)). Before the first dose of vaccine (day 1), patients will undergo blood sampling for immunological markers (quantification of circulating tumour-specific immune effectors) and a baseline DTH test. About 3 MU IL-2/day will be administered subcutaneously for 5 days starting the day after each vaccine dose. Vaccine doses will be injected intradermally into two sites near inguinal or axillary lymph node stations that were not sites of previous surgical exeresis. The first dose (week 1 (WK1)) will consist of freshly prepared vaccine, while cryopreserved aliquots will be used for all further doses. The remaining five doses will be administered every 4 weeks to complete 6 months of therapy (six vaccines). Tumour assessment, blood sampling for immunological biomarkers and DTH tests will be performed every 12 weeks starting from day 1 (first vaccine). In the event of a shortage of vaccine, patients will undergo additional leucapheresis on WK15. After six vaccine doses, patients will enter the follow-up phase.

\section{Follow-up arm and follow-up phase after vaccination}

Patients randomised to the follow-up arm and those who have completed the treatment phase will undergo laboratory and clinical assessment, tumour restaging and blood tests for immunological biomarkers every 12 weeks until progressive disease (PD) is confirmed and an alternative anticancer regimen initiated.

\section{Study population}

Patients disease free after surgery for metachronous stage III or stage IV melanoma.

\section{Inclusion criteria}

1. Signed written informed consent: patients must be willing and able to give written informed consent, and this must be done before entering the screening phase.

2. Availability of autologous tumour tissue fulfilling acceptance criteria specified in the 'Product Specification File'.

3. Histological or cytological confirmation of melanoma (any type of melanoma).

4. Disease-free status after surgical removal of metastatic lesions (stage IV or metachronous stage III). 
5. ECOG (easter cooperative oncology gorup) performance status $0-1$.

6. Negative screening tests for HIV, hepatitis B virus (HBV), hepatitis $\mathrm{C}$ virus (HCV) and syphilis $<30$ days before performing any of the GMP-regulated activities required (leucapheresis, collection of bioptic tumour material destined for tumour lysate/homogenate preparation).

7. Either sex, age $>18$ years.

8. An adequate method of contraception in women of childbearing potential must be using during the study and for up to 8 weeks after the study to minimise the risk of pregnancy.

9. Normal organ and marrow function.

\section{Exclusion criteria}

1. Positive testing to HCV, HBV, HIV or syphilis (specific blood tests $<30$ days before any GMP-regulated activity (leucapheresis, collection of bioptic tumour material destined for tumour lysate/homogenate preparation).

2. Prior lines of systemic chemotherapy, immunotherapy or biological therapy for metastatic melanoma.

3. Participation in another clinical trial with any investigational agents $<30$ days before study screening.

4. Uncontrolled intercurrent illness including, but not limited to, ongoing or active infection, symptomatic congestive heart failure, unstable angina pectoris, cardiac arrhythmia or psychiatric illness/social situations that would limit compliance with study requirements (as per the judgement of the treating physician).

5. Other known neoplastic diseases in the patient's medical history with a disease-free interval $<3$ years (except for previously treated basal cell carcinoma and in situ carcinoma of the uterine cervix).

6. Any contraindication to undergo leucapheresis, as evaluated by a blood transfusion service (eg, severe anaemia, thrombocytopaenia, oral anticoagulant therapy) or surgery.

\section{CLINICAL ENDPOINT ASSESSMENT}

\section{Types of recurrence}

Recurrence will be categorised as local, regional or distant. Local recurrence is defined as recurrence within the surgical area of the primary lesion. Regional (nodal) recurrence is defined as recurrence in the draining lymph nodes of the primary site of melanoma. Distant metastatic recurrence is defined as the spread of disease beyond the limits defined as local or regional recurrence.

\section{Relapse-free survival}

Definition of the date of disease recurrence: date on which a clinically suspicious lesion is first recorded in the patient's medical records, the action taken for which subsequently confirms a diagnosis of recurrence. Recurrence of disease can be locoregional, distant (metastatic) or a second primary malignancy. RFS is calculated as the time from enrolment/randomisation to either the date of disease progression or death. The date of first documented disease recurrence (if applicable) will be used as the date of event. Patients alive with NED at the time of their last follow-up visit will be censored at the time of their last clinical instrumental examination.

\section{Overall survival}

OS is calculated as the time from enrolment/randomisation to the date of death from any cause. Patients still alive at the time of data analysis will be censored at the last time they were known to be alive.

\section{IMMUNOLOGICAL ENDPOINT ASSESSMENT Immunological efficacy}

Immunological efficacy will be measured on the basis of the DTH test response to ATL and KLH after at least three vaccine doses.

\section{DTH test}

DTH testing is a classic method for measuring cellular immune reactivity. The technique involves intradermal administration of an antigen preparation and registration of the degree of erythema and induration produced 24-48 hours after the injection. This response reflects antigen-specific recruitment and activation of $\mathrm{CD} 4^{+}$to release T-helper 1 cytokines (in particular, interferon- $\gamma($ IFN- $\gamma$ )) and $\mathrm{CD}^{+}$effector T cells in the injection site. DTH testing will be performed in all patients on day 1 (pretreatment DTH, which showed no reactivity in the vast majority of patients evaluated in our previous studies), on the day before the fourth vaccine dose (post-treatment DTH) and at the end of treatment (after the sixth dose). Scalar doses $(100,50,20,10$ and $5 \mu \mathrm{g})$ of ATL and KLH (added to DCs together with tumour lysate as an adjuvant) will be used. The diameter of induration/erythema observed after 24 hours will be recorded according to the following scale: $0-5 \mathrm{~mm}$ grade $1,6-10 \mathrm{~mm}$ grade $2,11-20 \mathrm{~mm}$ grade 3 and $>21 \mathrm{~mm}$ grade 4 . Given that DTH reactivity to lower concentrations of the antigen(s) is closely related to more intense antigen-specific immune response, score results will be normalised against the concentration itself and transformed into a 0-80 scale for purposes of analysis. The best result obtained for each patient at any of the post-treatment DTH tests (whether for ATL or KLH) will be taken into account for data analysis (best normalised score). Patient DTH scores will also be evaluated in combination with the IFN- $\gamma$ ELISPOT analysis of circulating effectors.

\section{PATIENT AND PUBLIC INVOLVEMENT}

Patients or the public were not directly involved in the research. Nevertheless, the main results will be made available in the public domain.

\section{DISCUSSION}

Adjuvant therapy for resected high-risk melanoma is an area lacking in truly effective therapeutic strategies. 
Median RFS is $<5$ months and median OS ranges from 12 to 36 months. ${ }^{324}$ Similarly, a subset analysis of resected stage IV patients in the ECOG4697 study comparing GM-CSF with placebo showed a median DFS of 12 and 6 months, respectively. ${ }^{25}$ Patients with stage IIIC melanoma also have a poor prognosis but high-dose pegylated IFN- $\alpha 2 b$ is approved as adjuvant therapy for that specific subgroup in the US. ${ }^{126-28}$ The positive impact of ipilimumab on RFS, similar to that of high-dose IFN, has been sufficiently robust to garner Food and Drug Administration (FDA) approval. Taking into account that Weber $e t$ al recently reported that nivolumab improved RFS and showed lower toxicity rates than ipilimumab in resected stage IIIB, IIIC or IV melanoma, the use of that antibody in this setting will probably be abandoned ${ }^{29}$ However, many oncologists may be unwilling to prescribe an immune checkpoint inhibitor given the high incidence of irreversible toxicity (including serious autoimmune disease), an outcome that is difficult to accept in a population in whom as many as half may remain disease free without treatment. In the absence of an unambiguously positive approved agent for adjuvant therapy in melanoma, clinical trial participation remains a priority.

The most promising approaches currently under investigation include BRAF (v-raf murine sarcoma viral oncogene homolog B1) inhibitors and PD-1 pathway blockers. ${ }^{30}$ Although doubts remain about the ability of PD-1 blockers to effectively activate T cells when there is still non-measurable metastatic disease, it is hoped that these agents will improve on existing treatments (as in stage IV disease) and provide universally accepted adjuvant treatment approaches for high-risk melanoma. Results are eagerly awaited of the two adjuvant studies on targeted therapies, BRIM8 (vemurafenib alone) and COMBI-AD (dabrafenib+trametinib).

In conclusion, there are still no FDA-approved adjuvant therapeutic options for patients with resected stage IV melanoma. In our long-standing clinical experience, DC vaccination has maintained a very low toxic profile and has proven more effective in patients who develop a positive immune response to the in vivo DTH test. Furthermore, long-term survivors have not experienced the irreversible toxicity (eg, endocrine dysfunction, diabetes, pneumonia) that often accompanies treatment with immune checkpoint inhibitors. We hope to show that our very low toxicity DC-based vaccination immunotherapy can improve RFS and thus prolong OS.

\section{Confidentiality}

This study will be conducted in full conformity with relevant regulations and with ICH Guidelines for Good Clinical Practice (CPMP/ICH/135/95) July 1996, Directive 2001/20/EEC of the European Parliament, detailed guidelines on good clinical practice specific to advanced therapy medicinal products (ENTR/F/2/SF/dn D (2009) 35810), and other relevant current local legislation. Participants will be allocated a unique identification (ID) number at entry. The master list linking participant personal information and ID number will be maintained in a separate locked cabinet and password-protected hard drive. Data will be analysed by ID number only. Patient files and other source data will for be kept a maximum of 15 years.

\section{Dissemination}

Data deriving from this clinical trial are not intended for drug registration or for patent applications, but only for scientific and educational purposes which include presentation at scientific meetings, congresses and symposia and/or publication in scientific journals.

\section{Author affiliations}

${ }^{1}$ Immunotherapy Unit, Istituto Scientifico Romagnolo per lo Studio e la Cura dei Tumori (IRST) IRCCS, Meldola, Italy

${ }^{2}$ Unit of Biostatistics and Clinical Trials, Istituto Scientifico Romagnolo per lo Studio e la Cura dei Tumori I(RST) IRCCS, Meldola, Italy

${ }^{3}$ Advanced Oncological Surgery, Morgagni-Pierantoni Hospital, Forli, Italy

Acknowledgements The authors wish to thank Gráinne Tierney and Cristiano Verna for editorial assistance.

Contributors LR, FdR, FT, MF: conceived the idea for the project, developed the study protocol and drafted the manuscript. GG, ON: provided methodological support and designed a clinical information data extraction method for the protocol. LF, MP, AMG, VA, EP, VS, SC, JB, AR: are responsible for the production of the $\mathrm{DC}$ vaccine and for immunomonitoring during treatment. MG: revised the paper for important intellectual content. All authors approved the final version of the manuscript for submission.

Funding The authors have not declared a specific grant for this research from any funding agency in the public, commercial or not-for-profit sectors.

Competing interests None declared.

Patient consent Obtained.

Ethics approval Local Ethics Committee and the Italian Medicines Agency.

Provenance and peer review Not commissioned; externally peer reviewed.

Open access This is an open access article distributed in accordance with the Creative Commons Attribution Non Commercial (CC BY-NC 4.0) license, which permits others to distribute, remix, adapt, build upon this work non-commercially, and license their derivative works on different terms, provided the original work is properly cited, appropriate credit is given, any changes made indicated, and the use is non-commercial. See: http://creativecommons.org/licenses/by-nc/4.0/.

\section{REFERENCES}

1. Balch CM, Gershenwald JE, Soong SJ, et al. Final version of 2009 AJCC melanoma staging and classification. J Clin Oncol 2009;27:6199-206.

2. Essner R, Lee JH, Wanek LA, et al. Contemporary surgical treatment of advanced-stage melanoma. Arch Surg 2004;139:961-6. discussion 966-7.

3. Sosman JA, Moon J, Tuthill RJ, et al. A phase 2 trial of complete resection for stage IV melanoma: results of Southwest Oncology Group Clinical Trial S9430. Cancer 2011;117:4740-6.

4. Howard JH, Thompson JF, Mozzillo N, et al. Metastasectomy for distant metastatic melanoma: analysis of data from the first Multicenter Selective Lymphadenectomy Trial (MSLT-I). Ann Surg Oncol 2012;19:2547-55.

5. Wasif N, Bagaria SP, Ray P, et al. Does metastasectomy improve survival in patients with Stage IV melanoma? A cancer registry analysis of outcomes. J Surg Oncol 2011;104:111-5.

6. Stewart JH, Levine EA. Role of bacillus Calmette-Guérin in the treatment of advanced melanoma. Expert Rev Anticancer Ther 2011;11:1671-6.

7. Garbe C, Peris K, Hauschild A, et al. Diagnosis and treatment of melanoma. European consensus-based interdisciplinary guideline Update 2016. Eur J Cancer 2016;63:201-17. 
8. Singh B, Salama A. Updates in Therapy for Advanced Melanoma. Cancers 2016;8:17.

9. Kroon BB. Surgery for distant metastatic melanoma improves survival. Ann Surg Oncol 2012;19:2426-7.

10. Schuler G. Dendritic cells in cancer immunotherapy. Eur J Immunol 2010;40:2123-30.

11. Bol KF, Schreibelt G, Gerritsen WR, et al. Dendritic cell-based immunotherapy: state of the art and beyond. Clin Cancer Res 2016;22:1897-906.

12. Smyth MJ, Dunn GP, Schreiber RD. Cancer immunosurveillance and immunoediting: the roles of immunity in suppressing tumor development and shaping tumor immunogenicity. Adv Immunol 2006;90:1-50.

13. Whiteside TL. Inhibiting the inhibitors: evaluating agents targeting cancer immunosuppression. Expert Opin Biol Ther 2010;10:1019-35.

14. Whiteside TL. Disarming suppressor cells to improve immunotherapy. Cancer Immunol Immunother 2012;61:283-8.

15. Nestle FO, Alijagic S, Gilliet M, et al. Vaccination of melanoma patients with peptide- or tumor lysate-pulsed dendritic cells. Nat Med 1998;4:328-32.

16. Engell-Noerregaard L, Hansen TH, Andersen MH, et al. Review of clinical studies on dendritic cell-based vaccination of patients with malignant melanoma: assessment of correlation between clinical response and vaccine parameters. Cancer Immunol Immunother 2009;58:1-14.

17. Oshita C, Takikawa M, Kume A, et al. Dendritic cell-based vaccination in metastatic melanoma patients: phase II clinical trial. Oncol Rep 2012;28:1131-8.

18. Ridolfi L, Petrini M, Fiammenghi L, et al. Dendritic cell-based vaccine in advanced melanoma: update of clinical outcome. Melanoma Res 2011;21:524-9.

19. Guidoboni M, Granato AM, Ancarani V, et al. DC vaccination concurrently reduces Treg and anhances activated CTL in tumor biopsies from immunoresponsive patients with advanced melanoma. Abstract for the 26th annual scientific meeting of the society for immunotherapy of cancer [abstract]. J Immunotherapy 2011;34:670.

20. Ridolfi L, Petrini M, Granato AM, et al. Low-dose temozolomide before dendritic-cell vaccination reduces (specifically) CD4+CD25++Foxp3+ regulatory T-cells in advanced melanoma patients. J Trans/ Med 2013;11:135.

21. de Rosa F, Ridolfi L, Fiammenghi L, et al. Dendritic cell vaccination for metastatic melanoma: a 14-year monoinstitutional experience. Melanoma Res 2017;27:351-7.
22. Dillman RO, Cornforth AN, Depriest C, et al. Tumor stem cell antigens as consolidative active specific immunotherapy: a randomized phase II trial of dendritic cells versus tumor cells in patients with metastatic melanoma. J Immunother 2012;35:641-9.

23. Dillman RO, McClay EF, Barth NM, et al. Dendritic versus tumor cell presentation of autologous tumor antigens for active specific immunotherapy in metastatic melanoma: impact on long-term survival by extent of disease at the time of treatment. Cancer Biother Radiopharm 2015;30:187-94.

24. Hsueh EC, Essner R, Foshag LJ, et al. Prolonged survival after complete resection of disseminated melanoma and active immunotherapy with a therapeutic cancer vaccine. J Clin Oncol 2002;20:4549-54.

25. Lawson DH, Lee S, Zhao F, et al. Randomized, placebo-controlled, phase iii trial of yeast-derived granulocyte-macrophage colonystimulating Factor (GM-CSF) versus peptide vaccination versus gm-csf plus peptide vaccination versus placebo in patients with no evidence of disease after complete surgical resection of locally advanced and/or stage iv melanoma: a trial of the eastern cooperative oncology group-american college of radiology imaging network cancer research group (E4697). J Clin Oncol 2015;33:4066-76.

26. Eggermont AM, Suciu S, Santinami M, et al. Adjuvant therapy with pegylated interferon alfa-2b versus observation alone in resected stage III melanoma: final results of EORTC 18991, a randomised phase III trial. Lancet 2008;372:117-26.

27. Kirkwood JM, Ibrahim JG, Sosman JA, et al. High-dose interferon alfa- $2 \mathrm{~b}$ significantly prolongs relapse-free and overall survival compared with the GM2-KLH/QS-21 vaccine in patients with resected stage IIB-III melanoma: results of intergroup trial E1694/ S9512/C509801. J Clin Oncol 2001;19:2370-80.

28. Kirkwood JM, Manola J, Ibrahim J, et al. A pooled analysis of eastern cooperative oncology group and intergroup trials of adjuvant high-dose interferon for melanoma. Clin Cancer Res 2004;10:1670-7.

29. Weber J, Mandala M, Del Vecchio M, et al. Adjuvant Nivolumab versus Ipilimumab in Resected Stage III or IV Melanoma. N Engl J Med 2017;377:1824-35.

30. Eggermont AM, Chiarion-Sileni V, Grob JJ, et al. Adjuvant ipilimumab versus placebo after complete resection of high-risk stage III melanoma (EORTC 18071): a randomised, double-blind, phase 3 trial. Lancet Oncol 2015;16:522-30. 